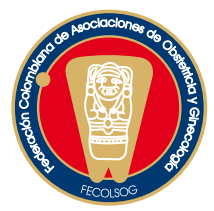

SERIE DE CASOS

\title{
TROMBOSIS DE SENO VENOSO CEREBRAL Y POSIBLE ASOCIACIÓN CON ANTICUERPOS ANTINUCLEARES. REPORTE DE DOS CASOS Y REVISIÓN DE LA LITERATURA
}

\section{Thrombosis of cerebral venous sinuses and possible association with antinuclear antibodies. A report of two cases and a review of the pertinent literature}

Hernán Cortés Y., M.D.*, Mauricio Herrera M., M.D.**, Mario Rebolledo A., M.D.***

Recibido: junio 28/07 - Aceptado: febrero 20/08

\section{RESUMEN}

Se presentan dos pacientes posparto con antecedente de preeclampsia, que consultaron por cefalea y síntomas neurológicos, en quienes se diagnosticó trombosis de los senos venosos cerebrales, con una posible asociación a presencia de anticuerpos antinucleares.

Se hace una revisión de la literatura de la trombosis de los senos venosos durante el posparto con énfasis en el diagnóstico y las recomendaciones del tratamiento con anticoagulantes.

Se recomienda la terapia anticoagulante por tres a seis meses. En ambas pacientes descritas se presentó un aumento de los títulos de anticuerpos antinucleares, por lo que surge la hipótesis de una posible asociación causal.

Palabras clave: trombosis, seno venoso cerebral, anticoagulación, resonancia magnética, embarazo, puerperio.

* $\quad$ Fellow Medicina Materno Fetal. Universidad del Rosario. Docente Departamento de Obstetricia y Ginecología U de A. Unidad de Medicina Materno Fetal, Clínica Colsanitas. Bogotá, Colombia. Correo electrónico: hcortes@medicina.udea.edu.co

** Especialista en Medicina Materno Fetal. Jefe del programa de Fellow Clínica Colsanitas. Unidad de Medicina Materno Fetal. Bogotá, Colombia.

*** Especialista en Medicina Materno Fetal. Docente del programa de Fellow Clínica Colsanitas. Unidad de Medicina Materno Fetal. Bogotá, Colombia.

\section{SUMMARY}

Two postpartum patients, having a history of preeclampsia, were presented. They had consulted because of cephalalgia and neurological symptoms being presented; the diagnosis was thrombosis of the cerebral venous sinuses, possibly associated with the presence of antinuclear antibodies.

The literature regarding thrombosis concerning venous sinuses during the postpartum period was reviewed, placing special emphasis on diagnosis and instructions for treatment with anticoagulants.

Three to six months' anticoagulant treatment is recommended. Both patients presented increased antinuclear antibody titres, suggesting possible causal association.

Key words: thrombosis, cerebral venous sinus, anticoagulant therapy, magnetic resonance image, pregnancy, puerperium.

\section{INTRODUCCIÓN}

La trombosis del seno venoso cerebral es una complicación rara de la gestación que se puede presentar durante el embarazo y especialmente en el puerperio.

Es importante tener este diagnóstico en mente como diagnóstico diferencial, ya que puede ser 
pasado por alto en pacientes con cefalea persistente, debido a que no hay síntomas ni signos patognomónicos. El pronóstico varía según el sitio de los reportes publicados.

El objetivo de la presentación es hacer una revisión de la literatura sobre trombosis de los senos venosos posparto con énfasis en el diagnóstico y las recomendaciones del tratamiento con anticoagulantes.

\section{Caso 1}

Mujer de 34 años, gestaciones, 3; partos, 2; aborto, 1; un hijo vivo; sin antecedentes previos relevantes, que presentó un parto vértice espontáneo de un feto muerto de 22 semanas, asociado a la hipertensión gestacional, sin proteinuria (valor $160 \mathrm{mg}$ en orina de 24 horas), fue dada de alta dos días después con tratamiento antihipertensivo (captopril). En el cuarto día posparto consulta nuevamente al servicio de urgencias por cefalea occipital severa, que no mejora con manejo analgésico, asociado a visión borrosa ocasional y náuseas.

$\mathrm{Al}$ examen físico, se encuentra con una presión arterial de 190/120, pulso de $80 \mathrm{x}$ minuto, útero bien contraído y escasos loquios, sin edemas y un examen neurológico que se describe como normal.

Se hospitaliza con diagnóstico de preeclampsia severa posparto y se inicia manejo con sulfato de magnesio, se aumenta la dosis del antihipertensivo, analgesia, hidratación intravenosa y se solicitan paraclínicos que se reportan como normales.

La paciente continúa con la cefalea severa a pesar del tratamiento, por tal motivo se solicita una angioresonancia magnética, por sospecha de enfermedad cerebrovascular.

La resonancia demuestra trombosis del seno transverso derecho, con ausencia de flujo a yugular interna ipsilateral, sin lesiones hemorrágicas adyacentes. Figuras 1 y 2

La paciente es valorada por la unidad de medicina materno fetal y el servicio de neurología, se inicia anticoagulación con enoxaparina subcutánea $60 \mathrm{mg}$ cada 12 horas y warfarina $5 \mathrm{mg}$ /día, se solicitan, además, estudios para trombofilia; la paciente evoluciona satisfactoriamente y es dada de alta al quinto día con warfarina (INR: 2,3) y revisión ambulatoria por la unidad y neurología.

Los estudios para anticuerpo antifosfolípido, factor V Leiden y anti DNA fueron negativos, los anticuerpos antinucleares se reportaron en 1/320 (valores significativos mayor a 1/80).

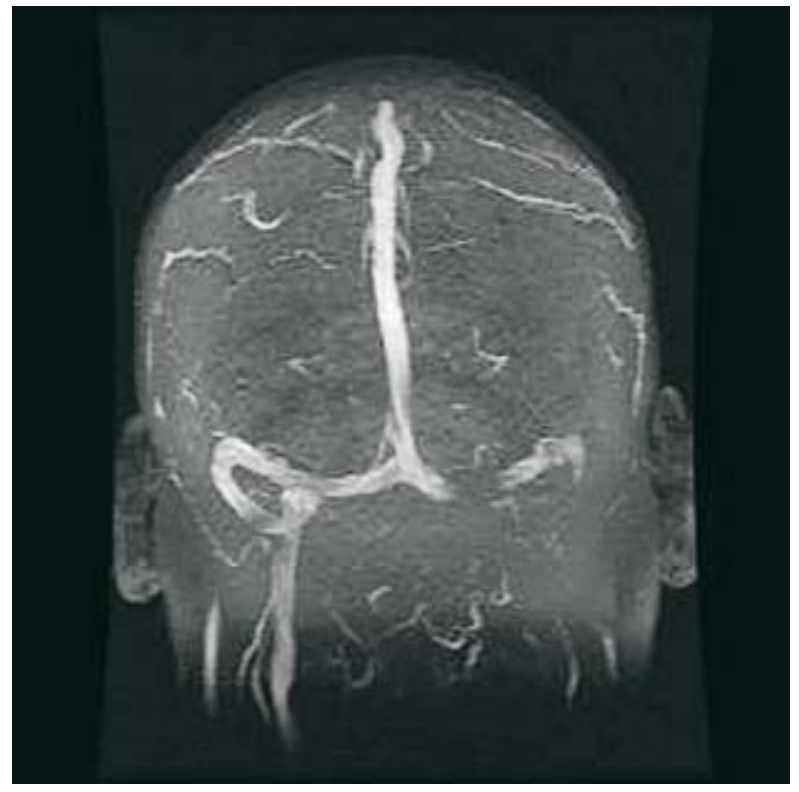

Figura 1.

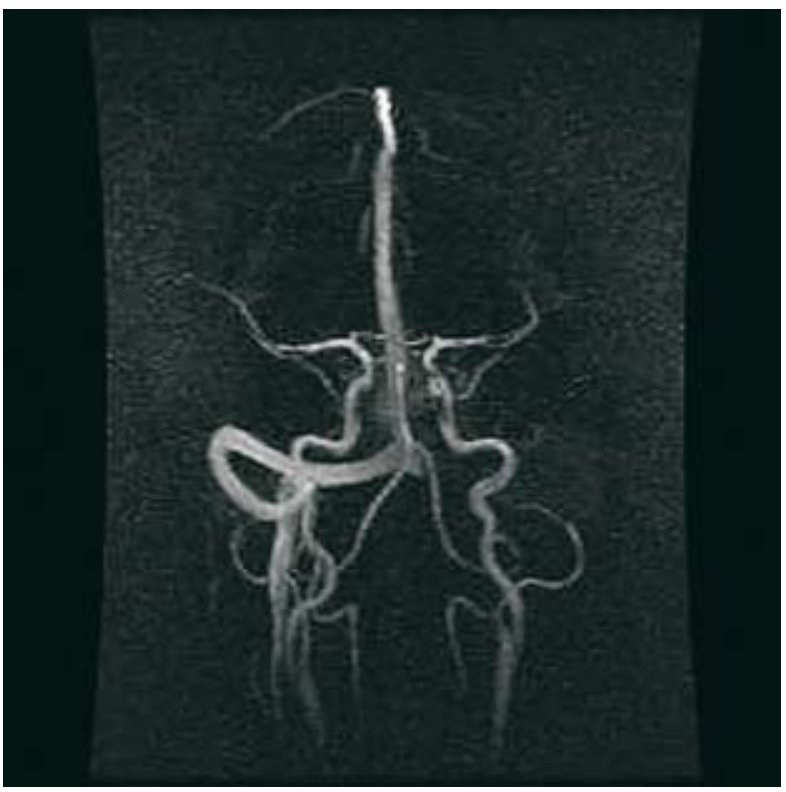

Figura 2. Nótese la imagen de defecto en el seno transverso derecho y ausencia de flujo en la yugular interna ipsilateral (derecha). 


\section{Caso 2}

Mujer de 29 años, primigestante, sin antecedentes previos relevantes, con embarazo de 28 semanas, a quien se le realizó cesárea urgente por eclampsia, es dada de alta siete días después con laboratorios normales y manejo antihipertensivo ambulatorio con nifedipina y captopril.

Consulta nuevamente a los diez días posparto, por un cuadro de inicio súbito de parestesias y hemiparesia derechas, que le imposibilitan la marcha, asociado a cefalea frontal que no mejora con analgesia.

$\mathrm{Al}$ examen físico se encuentra con una presión arterial de 120/80, pulso: 70 x minuto, un útero bien contraído y herida sana, al examen neurológico se evidencia una parálisis facial central derecha, disminución de la fuerza muscular de hemicuerpo derecho de predominio en miembro superior, sensibilidad normal, reflejos normales y respuesta plantar flexora bilateral.

Con diagnóstico de enfermedad cerebrovascular, se solicita angio-resonancia magnética, que reporta trombosis del seno longitudinal superior y edema cortical focal fronto-parietal izquierdo. Figura 3

Se inicia anticoagulación con enoxaparina $60 \mathrm{mg}$ subcutáneo cada 12 horas y warfarina $5 \mathrm{mg}$ /día, se solicitan además estudios para trombofilia; la paciente evoluciona satisfactoriamente y es dada de alta siete días después con resolución de los síntomas neurológicos, en tratamiento con warfarina y un INR de 2,5 con revisión ambulatoria por neurología.

La proteína S se reportó en 31\%, por debajo de lo esperado aun en mujeres en embarazo (valores normales de 60-118\%) y anticuerpos antinucleares de 1/1.280; los estudios para anticuerpos antifosfolípido, factor $\mathrm{V}$ Leiden, proteína $\mathrm{C}$, antitrombina y mutación del gen protrombina 20210A, se reportaron como normales.

\section{DISCUSIÓN}

El embarazo aumenta el riesgo de tromboembolia cinco veces en comparación con las personas no embarazadas y el riesgo es mayor durante el puerperio;

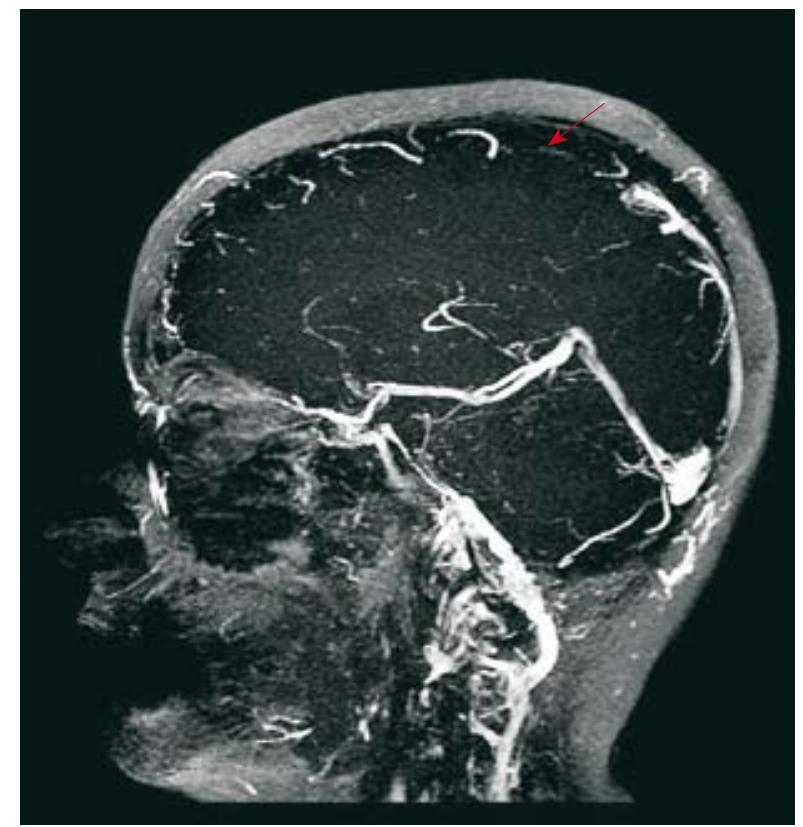

Figura 3. La flecha señala la imagen de defecto del seno longitudinal superior.

la incidencia de la enfermedad tromboembólica en las pacientes obstétricas varía de 0,06\% a 1,8\% y múltiples factores aumentan este riesgo, a saber: la cesárea, la preeclampsia, la obesidad, la gestación múltiple, la multiparidad y la obesidad asociada, entre otras.

Una de las manifestaciones de la enfermedad troboembólica en la embarazada es la trombosis del seno venoso cerebral (TSVC), que tiene una incidencia estimada entre 10 a 20 por 100.000 embarazos, ${ }^{2}$ se asocia con la preeclampsia, el parto por cesárea, deshidratación, presencia de infecciones y trombofilias. El 70 - 80\% de los casos se desarrollan durante el puerperio. ${ }^{3}$

Los síntomas más comunes son: la cefalea (95\%), convulsiones (47\%), paresia (43\%) y papiledema (41\%), pero también se puede manifestar con letargo y coma; ${ }^{4}$ en una serie se describe que el $25 \%$ de los pacientes tenían un examen neurológico normal al momento del diagnóstico. ${ }^{5}$

En un estudio realizado por Stella y colaboradores, se encontró que el 15\% de las pacientes que consultaban por cefalea posparto, tenían un hallazgo anormal en los estudios de imagenología, con TSVC en el 3\% de los casos. ${ }^{6}$ 
El seno transverso es el más frecuentemente afectado, como ocurrió en la primera paciente descrita, seguido por los senos venosos sagitales y sigmoideo, ${ }^{5}$ no se describen diferencias entre el lado derecho o izquierdo.

El diagnóstico se puede sospechar en la tomografía cerebral con contraste, por la presencia de infartos a menudo asociados a zonas de hemorragia y por la presencia de defectos de llenado en la parte posterior del seno sagital (signo delta vacío), normalmente, el seno venoso se visualiza como un triángulo blanco en la tomografía por contraste. La resonancia con venografía, (como la realizada en estas pacientes, figuras 1-3) o la angiografía cerebral son diagnósticas. ${ }^{4}$ Se recomienda además realizar tamizaje para trombofilias, pues estas se presentan hasta en un 20\% de los casos, siendo las más comúnmente encontradas en orden descendente, el síndrome de anticuerpos antifosfolípido, la deficiencia de proteínas $\mathrm{C}$ y $\mathrm{S}$, la deficiencia de antitrombina y el factor V Leiden; ${ }^{5,7}$ llama la atención que en estos dos casos se presentó un aumento significativo de los anticuerpos antinucleares, pero en las bases de datos revisadas (OVID, MD Consult y Medline) no se encontró ninguna referencia que asocie este hallazgo (de manera aislada sin presencia de lupus) con el desarrollo de trombosis. De igual manera, es posible que la segunda paciente tenga una deficiencia de proteína $\mathrm{C}$, la cual queda por confirmar cuando se suspenda la anticoagulación.

Una vez establecido el diagnóstico, se debe iniciar la anticoagulación con heparina no fraccionada o heparinas de bajo peso molecular, durante la fase aguda, aun en presencia de infarto hemorrágico asociado y continuar después con warfarina por tres a seis meses, manteniendo el INR entre 2 y 3. En los pacientes que presentan un deterioro neurológico progresivo, a pesar de una adecuada anticoagulación, se recomienda la terapia trombolítica.$^{8}$ En caso de trombofilia, algunos autores sugieren continuar la anticoagulación por tiempo indefinido, principalmente, si el evento ocurrió sin un factor desencadenante claro. ${ }^{8}$ En las pacientes a quienes se les diagnosticó déficit de antitrombina, síndrome antifosfolípido, factor V Leiden o mutación del gen protrombina 20210A, se recomienda la anticoagulación durante el embarazo y el puerperio. En caso de otras trombofilias, como el déficit de proteína $\mathrm{S}$ que posiblemente presenta la segunda paciente que se reportó, se recomienda profilaxis durante el embarazo y anticoagulación posparto. ${ }^{9-11}$

El pronóstico en general es bueno, con una mortalidad del $8 \%$ y secuelas severas en el 2,2\% de los pacientes luego de un año ${ }^{8}$; sin embargo, otros estudios en países en desarrollo reportan una mortalidad de la TSVC asociada al embarazo cercana al 30\%, debido, posiblemente, a un diagnóstico tardío o compromiso de la enfermedad de base. ${ }^{12}$ En pacientes no embarazadas los principales factores que se asocian con el riesgo de mortalidad son la edad mayor de 37 años, la presencia de cáncer como factor desencadenante y la presencia de coma o hemorragia en el momento del diagnóstico. ${ }^{9}$ En un estudio se comparó la mortalidad por trombosis venosa profunda versus la mortalidad por TSVC, encontrando una tasa del 6,2\% y del 2,8\% respectivamente, atribuyendo la mayor mortalidad del primer grupo a una mayor edad en estos. ${ }^{5} \mathrm{Se}$ estima que el riesgo de recurrencia es de 2,2\% por año. No se han descrito recurrencias de la TSVC en posteriores embarazos.

\section{REFERENCIAS}

1. McLeod A, Ellis C. Prevention and Treatment of Venous Thromboembolism in High Risk Situations in Pregnancy. Fetal Mat Med Review 2005;16:51-70.

2. Lockhart EM, Baysinger CL. Intracranial venous thrombosis in the parturient. Anesthesiology 2007;107:652-8.

3. Lanska DJ, Kryscio RJ. Risk factors for peripartum and postpartum stroke and intracranial venous thrombosis. Stroke 2000;31:1274-82.

4. Karnad DR, Guntupalli KK. Neurologic disorders in pregnancy. Crit Care Med 2005;33:S362-71.

5. Gosk-Bierska I, Wysokinski W, Brown RD Jr, Karnicki K, Grill D, Wiste H, et al. Cerebral venous 
sinus thrombosis: Incidence of venous thrombosis recurrence and survival. Neurology 2006;67:814-9.

6. Stella CL, Jodicke CD, How HY, Harkness UF, Sibai BM. Postpartum headache: is your work-up complete? Am J Obstet Gynecol 2007;196:318.e1-7.

7. Shehata HA, Okosun H. Neurological disorders in pregnancy. Curr Opin Obstet Gynecol 2004;16: 117-22.

8. Albers GW, Amarenco P, Easton JD. Antithrombotic and thrombolytic therapy for ischemic stroke: the Seventh ACCP Conference on Antithrombotic and Thrombolytic Therapy. Chest 2004;126:483S-512S.

9. Ferro J.M, Canhão P, Stam J, Bousser MG, Barinagarrementeria F; ISCVT Investigators. Prognosis of cerebral vein and dural sinus thrombosis: results of the
International Study on Cerebral Vein and Dural Sinus Thrombosis (ISCVT). Stroke 2004;35:664-70.

10. Mehraein S, Ortwein H, Busch M, Weih M, Einhäupl $\mathrm{K}$, Masuhr F. Risk of recurrence of cerebral venous and sinus thrombosis during subsequent pregnancy and puerperium. J Neurol Neurosurg Psychiatry 2003;74:814-6.

11. Bates SM, Greer I, Hirsh J and Ginsberg JS. Use of antithrombotic agents during pregnancy: the Seventh ACCP Conference on Antithrombotic and Thrombolytic Therapy. Chest 2004;126:627S$644 \mathrm{~S}$.

12. Karnad DR, Lapsia V, Krishnan A, Salvi VS. Prognostic factors in obstetric patients admitted to an Indian intensive care unit. Crit Care Med 2004;32:1294-9.

\section{Conflicto de intereses: ninguno declarado}

